\title{
How to eat on the go
}

Dendritic cells use components of their cytoskeleton to both move and ingest pieces of infected cells. This competition for protein resources can give rise to a complex set of states that may be understood with an advection-diffusion model.

\section{Herbert Levine}

C ellular biology is frugal, often using the same protein building block in multiple independent functional constructs. What happens when these needs conflict? Writing in Nature Physics, Ido Lavi and colleagues ${ }^{1}$ have shown that competition for a key effector molecule, namely myosin II, can drive complex unexpected behaviour in the motion of a certain class of white blood cell. Moreover, the success of their model in explaining the data shows that the community of scientists applying physics to cell motility is rapidly progressing in its quantitative capabilities.

Eukaryotic cell motility, requiring the coordination of forces arising from the non-equilibrium physics of the cytoskeleton, continues to challenge physicists and biologists alike. The basic components have been clear for some time, with polymerized actin playing a primary role. Actin filaments can alter their spatial self-organization depending on their binding partners ${ }^{2}$. In many motile cell types, this leads to branched protruding structures at the front and contracting bundles at the rear ${ }^{3}$. How these possibilities combine with cytosol flow, adhesion to the substrate, and membrane mechanics to generate the myriad observed behaviours is the crux of what the field is striving to understand.

One of these critical binding partners is the protein myosin, a well-studied component of heart and skeletal muscle. Myosin acts as a molecular motor, converting chemical energy to perform work on actin filaments ${ }^{4}$. This mechanical action leads to active stresses inside the cell, generating actin flow (a net backward motion of the filament network in the frame of the cell) and ultimately helping to drive the cell forward. Self-consistently, myosin is driven to the rear of the cell by the actin flow and can be detected there by the tools of modern biological imaging; it is a primary contributor to the polarization of the cell into front and back regions. Several groups have created mathematical models of this flow ${ }^{5-7}$ and its consequences for cell polarity, with applications to issues such as motion persistence and the morphology of fish keratocytes.

But cells - including those that are motile - have a variety of essential tasks that they have to perform. Take for example the case of dendritic cells. A dendritic cell is a specialized form of white blood cell that plays an important role in antigen presentation. When faced with a foreign invader, the human immune system must learn to recognize the signs of an infected cell. One way this occurs is that dendritic cells 'swallow' pieces of infected cell debris and display protein fragments from this debris on the surface ${ }^{8}$. This display instructs lymphocytes (specifically T-cells) to search for this pattern in the nearby tissue and kill the offending presenters. For our purposes, the key notion is that this process requires much of the same polymeric machinery as does the motility engine. This type of dual use has been known for some time (and has recently ${ }^{9}$ been re-emphasized) in the simple Dictyostelium amoeba, where the swallowing is actually used for nutrition. However, the consequences, if any, of this possible resource conflict were unclear.

Lavi et al. ${ }^{1}$ re-analysed data ${ }^{10}$ on the motion of a dendritic cell through a microfabricated channel. The channel essentially removed most of the freedom of the cell to adopt different shapes and hence reduced the problem to tracking the net motion as a consequence of the variation of cytoskeletal components in the one spatial dimension. The experiment clearly indicated a critical role for myosin in determining the motion. Lavi et al. ${ }^{1}$ devised an advection-diffusion equation for myosin, coupled to a simple phenomenological equation spelling out how myosin determines motion. Their major assumption was that there is a dynamic balance between myosin in the rear part of the cell where it is used to power motion and myosin in the forward part where it allows for the membrane reorganization needed in antigen capture.

They then proceeded to analyse their nonlinear model with the tools of bifurcation theory, finding a Hopf bifurcation to an oscillating velocity state and also a homoclinic bifurcation to a state with periodic cell reversals. The resultant dynamical behaviour allowed them to make sense of the experimental data, which also exhibits rather complex trajectories. In fact, the agreement is quite outstanding, lending credence to their approach and indeed to the entire macroscopic modelling framework on which it is based.

Of course, this work is not the end of the story. Restriction to one-dimensional behaviour is a convenient protocol for experimenter and theorist alike, but is hardly the most physiological setting. It would thus be interesting to redo the experiment on two-dimensional surfaces and, eventually, inside realistic tissue microenvironments. A corresponding (and highly non-trivial) extension of the analysis would then be necessary. And we should not lose sight of the fact that the parameters chosen to make the model match the data are not directly measurable, but instead must be fit; this is a shortcoming of all cell motility models and prevents theory from being fully predictive. These caveats aside, however, it is exciting to see how far we have come in our ability to unravel one of the most interesting capabilities of living cells.

Herbert Levine is in the Department of Bioengineering at Rice University, Houston, Texas 77030, USA. e-mail: herbert.levine@rice.edu

\section{References}

1. Lavi, I. et al. Nature Phys. 12, 1146-1152 (2016).

2. Pollard, T. D. \& Borisy, G. G. Cell 112, 453-465 (2003).

3. Danuser, G., Allard, J. \& Mogilner, A. Annu. Rev. Cell Dev. Biol. 29, 501-528 (2013).

4. Warrick, H. M. \& Spudich, J. A. Annu. Rev. Cell Biol. 3, 379-421 (1987).

5. Rubinstein, B., Fournier, M. F., Jacobson, K., Verkhovsky, A. B. \& Mogilner, A. Biophys. J. 97, 1853-1863 (2009).

6. Shao, D., Levine, H. \& Rappel, W.-J. Proc. Natl Acad. Sci. USA 109, 6851-6856 (2012).

7. Maiuri, P. et al. Cell 161, 374-386 (2015).

8. Heuzé, M. L. et al. Immunol. Rev. 256, 240-254 (2013).

9. Veltman, D. M. Biochem. Soc. Trans. 43, 129-132 (2015).

10. Chabaud, M. et al. Nature Commun. 6, 7526 (2015)

Published online: 8 August 2016 